\title{
Vertical Handoff Optimization in 4G Heterogeneous Networks
}

\author{
Alka Kamra \\ Student, PDMCE
}

\begin{abstract}
Wimax and Wifi are the telecommunication technologies that offer transmission of wireless data via a number of transmission methods such as portable or fully mobile internet access via point to multipoint links. As the size of a Wireless Network is much vast the complete network is divided into clusters. Each cluster has a cluster head that controls all nodes of the network. These networks can have a node with the mobility. The problem begins when a node moves from the cluster to outside the range of a Cluster head. In such case, the control of that node is shifted to other Cluster Head. This process is called handoff mechanism. The proposed system is the scheme to select the best cluster head for this node respective to the reliability and efficiency. The proposed work is the selection of cluster head in case of heterogeneous network. Here the heterogeneity is defined in terms of two different networks these are Wimax and Wifi Networks. The process of handover between these networks is called vertical handover. This paper tells us how to minimize the vertical handover. In this work we have done the basic changes while performing the selection of base station.
\end{abstract}

Keywords: Wimax, Wifi, Cluster, Cluster Head, Handoff Mechanism, Heterogeneous Networks.

\section{I.INTRODUCTION}

Next-generation wireless networks have been envisioned as an Internet Protocol (IP) based infrastructure with the integration of various wireless access networks such as IEEE 802.11 wireless local area networks (WLANs), IEEE 802.16 wireless metropolitan area networks (WMANs), Wimax, Wi-Fi networks and Universal Mobile Telecommunications System (UMTS). Heterogeneous wireless networks should be made in a such a way that it should provide users an ubiquitous environment with seamless mobility and good quality of service (QoS). Mobile nodes (MNs) can automatically switch the connectivity between different types of networks.

The interworking between different wireless access networks has been an important research topic in the past few years. Different radio access technologies present distinct characteristics in terms of mobility management, security support, and QoS providing. For getting flawless mobility and end-to-end QoS guarantee for the users, these problems should be carefully taken care off while developing the interworking and handoff schemes of WMNs with various wireless networks. Mesh routers in the WMNs play an important role. The 802.11 access point (AP) functions and 802.16 base station (BS) functions can be integrated into one mesh router. In case the MN switches the network interface, only the type of link is different between the MN and mesh routers, and the MN still connects to the same mesh router. In this case, the traditional mobility management such as Mobile IP leads to a large handoff delay with too much signalling cost. Thus to achieve fast and seamless handoff, a new handoff scheme should be considered. Another factor which can effortlessly choose vertical handoff is how and when to make a handoff decision. In traditional handoff, the received signal strength is the main handoff metric. However, in vertical handoff, only the received signal strength is not enough to make a handoff decision. The handoff metrics may be cost of service, load on network, MN's distance, QoS and user preference. The vertical handoff may not take place only at the cell edge. It can occur at any time (even when the MN does not move) depending on the network condition and network congestion. Making a decision to activate a vertical handoff according to the system performance and QoS parameters becomes the important part of this particular kind of vertical handoff. Therefore an effective and efficient vertical handoff decision algorithm in the interworking between 802.11 and 802.16 in WMN is needed to maximize the utilization of resources and to avoid unnecessary handoff. In this work we have done the parametric changes while performing the selection of base station. The analysis is performed respective to the effective throughput and the delay.

\section{II.WIRELESS NETWORKS}

Wireless means transmitting signals using radio waves. The development of Wireless networks is more suitable for users People are giving more importance to the connections made of heterogeneous wireless networks. Universal wireless communication system consists of three layers of coverage and coverage decreases from top to bottom. Topmost layer consist of satellite system with global coverage. Middle layer consist of cellular network which covers metropolitan areas and bottom layer with LAN networks which cover hot spots. A common IP based network will 
connect them to each other. Hence multimode terminals required with seamless inter system vertical handover. Wireless networks are basically designed to do simple tasks such as switching off the television or doing complex tasks as supplying the sales force with information from an automated enterprise application.

\section{WIMAX TECHNOLOGY}

WiMAX means Worldwide Interoperability for Microwave Access. This is a telecommunication technology that offers transmission of wireless data through number of transmission methods namely portable or fully mobile internet access via point to multipoint links. The WiMAX technology does not need any cable infrastructure and offers around 72 Mega Bits per second. WiMAX technology is based on Standard that is IEEE 802.16, it is usually called as Broadband Wireless Access. WiMAX technology is actually based on the standards that are making the possibility to deliver last minute broadband access as a substitute to conventional cable and DSL lines.

\section{Features of WiMAX}

WiMAX has a rich set of technological improvements compare to the other broadband access technology. The set of features of WiMAX are listed below:

- OFDM based physical layer: WiMAX is based on orthogonal frequency division multiplexing that offers multipath resistance and allow NLOS communication.

- High data rate: WiMAX can support very high peak data rate which is as high as 74 mbps.

- Quality of service: WiMAX MAC layer is responsible for QoS. WiMAX MAC layer support real time, non real time and best effort data traffic and its high data rate, sub channelization, and flexible scheduling improve the QoS.

- Flexible architecture: WiMAX architecture is very flexible. It can support point to point and point to multipoint connection according to its requirements. It also supports IP-based architecture that is easily converge with other networks and takes advantage of application development from the existing IP based application.

- TDD and FDD support: WiMAX support both time division duplex and frequency division duplex which helps in spectrum management, transceiver design and low cost system development.

- Adaptive modulation and coding: Adaptive modulation and coding scheme can connect more users. It is a technique to maximize throughput and able to setup connection in a low signal strength and noisy environment.

- Mobility support: WiMAX offer optimized handover which support full mobility application such as voice over internet protocol (VOIP). It has also the power saving mechanism which increases the battery life of handheld devices.

- Strong Security: WiMAX support extensible security feature for reliable data exchange. It use Advanced Encryption Standard (AES) encryption for secure transmission and for data integrity, it use data authentication mechanism.

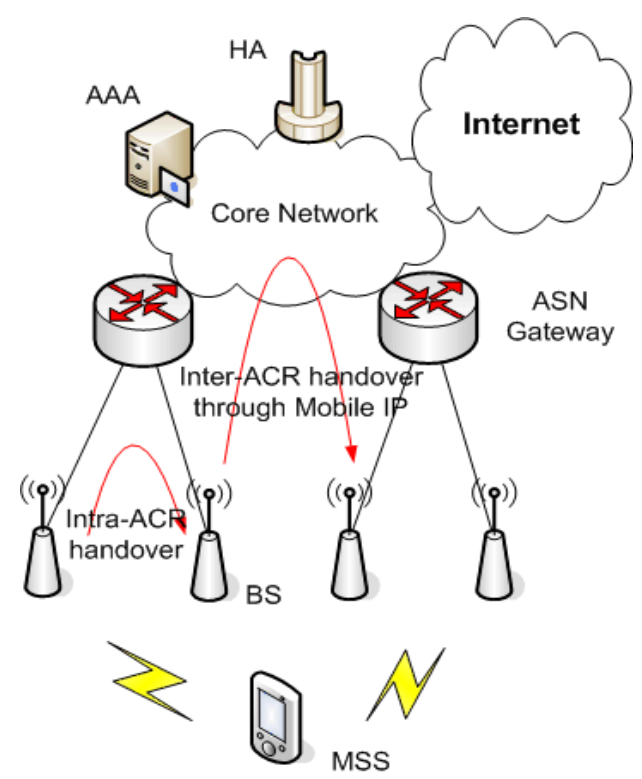

Fig 1 Network Architecture of WIMAX

Architecture of mobile WiMAX network consists of four main components: MSS, BS, Access Service Network gateway, and one core network. 
Vol. 6, Issue 6, June 2017

\section{IV.WI-FI TECHNOLOGY}

Wireless Fidelity (Wi-Fi) is a wireless technology which provides internet connectivity or connectivity among the users. In 1997 IEEE provide a set of specification and standards for Wi-Fi which is under the title 802.11 that explains the structure of the comparatively short range radio signal for Wi-Fi service. Many specifications came and most commonly used specifications are 802.11b, 802.11g and 802.11a. Out of these three, 802.11a can provide higher speeds within the various radio frequencies. IEEE is now working for a new standard $802.11 \mathrm{n}$ which is more reliable, secure and faster than the other standard. Wi-Fi was created for wireless extension for the wired LAN. Due to this reason the distance between the $\mathrm{Wi}$-Fi access point and user equipment is limited to around 100 feet indoor and up to 300 feet outdoors. So if a user moves its computer to a new location, he/she should find a new access point for continuing the communication. Due to the worldwide availability of the equipment and its less maintenance and servicing cost, Wi-Fi is widely accepted throughout the world and it is widely used in a restaurants, hotels, airports and school campuses. It is also work well in the auditoriums, meeting rooms and small businesses. Internet service providers also use it for individual home connectivity and connectivity to the commercial complexes.

\section{Wi-Fi services}

Wi-Fi standard $802.11 \mathrm{~b}, 802.11 \mathrm{~g}$ and $802.11 \mathrm{n}$ operate in a $2.4 \mathrm{GHz}$ unlicensed frequency band. It's another standard 802.11 a uses $5.3 \mathrm{GHz}$ band for transmitting the data. This signal strength can provide the service from 40-100 meter which can cover the entire house and reducing the data rate further increase the coverage area. Moreover installing additional access point (AP) and increasing the output power level greatly improved the coverage area.

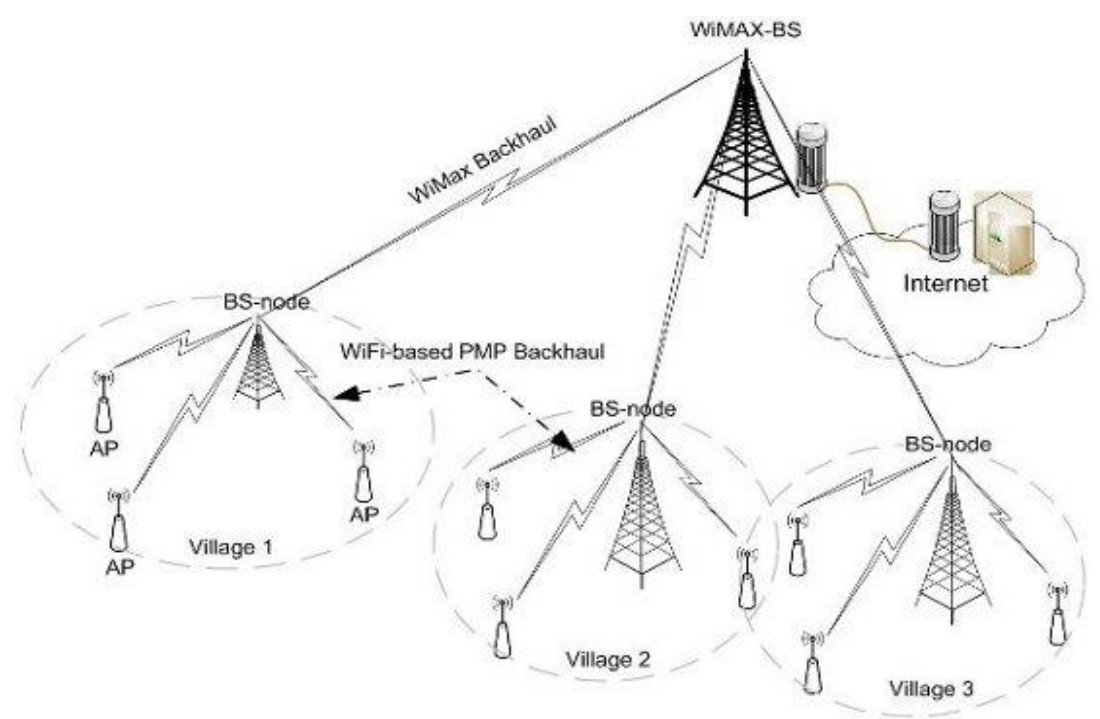

Fig 2 Network Architecture of Wifi

\section{HANDOFF- VERTICAL AND HORIZONTAL}

In next generation of wireless networks, mobile users can move between heterogeneous networks, using terminals with multiple access interfaces. The most important issue in such an environment is always best connection. To answer best connection requirement, various vertical handover decision strategies have been proposed in literature recently.

Vertical handoff is more complex than horizontal handoff. In traditional horizontal handoff, the main handoff metric is the received signal strength. How- ever, in vertical handoff, only the received signal strength is not enough to make a handoff decision. The handoff metrics may include cost of service, available band- width, power requirements, quality of service, and user preference. The place for vertical handoff is not at the edge of the cell, it can take place anywhere. It can take place anytime anywhere, depending on the network condition and user preference. Such combined metrics lead to a challenge for designing a handoff decision algorithm because some factors are more difficult to obtain than the physical layer parameters such as received signal strength (RSS) and signal-to-interference ratio.

Next Generation or $4 \mathrm{G}$ network can be achieved through the interworking of several existing architectures to form a seamless global network. An important issue involved in interworking is vertical handover. The issue of vertical handover must be addressed to achieve this integration. A handover occurs when an end user changes its point of attachment to a network. The nature of this point of attachment depends on the network architecture. For instance an end user in an 802.11 network attaches to an access point, while an end user in a GSM network attaches to a base station. Horizontal handover occurs when an end user moves between networks of the same and establishes a 
connection with a similar point of attachment, while vertical handover occurs when an end user changes its point of attachment from one network to another architecturally different network.

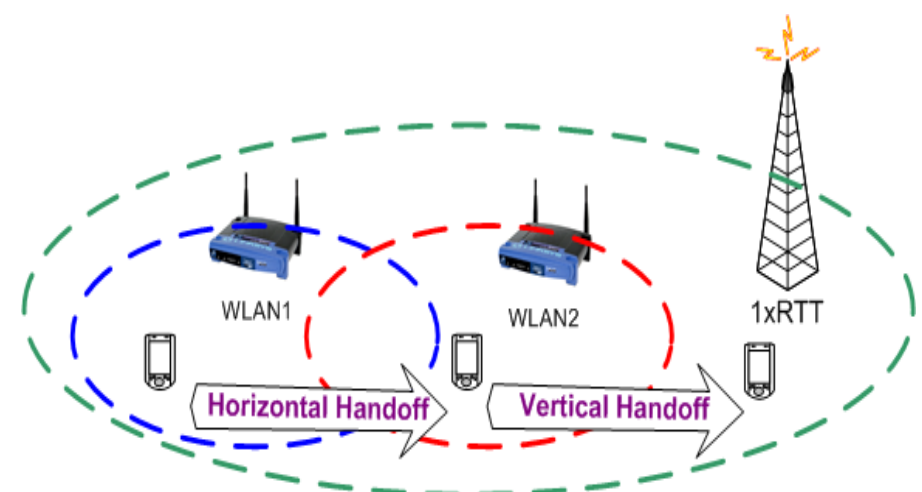

Fig 3 Horizontal vs Vertical Handoff

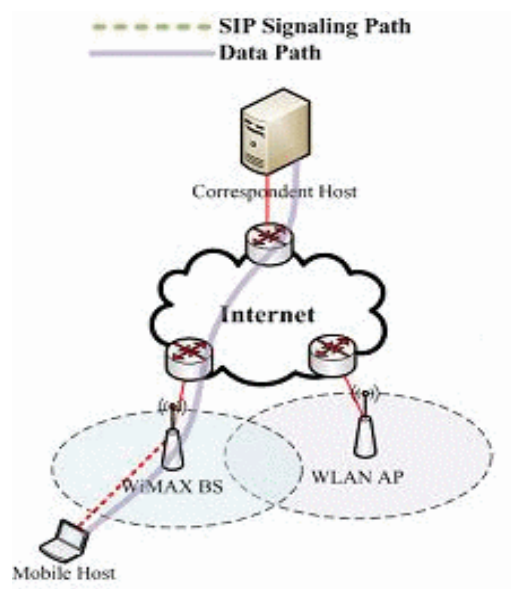

Fig 4 Vertical Handoff

\section{VI.CONCLUSION}

Hybrid network architecture supports all the models that are fixed, mobile \& nomadic. It is also support high capacity real time and non-real time voice, data and multimedia services while maintaining the appropriate QoS. It also supports idle mode operation and paging for the mobile station. Its network reference model support interoperability.

By comparing the Proposed Handover Approach and Existing and, we observed that Proposed Handover Approach offers better services than the Existing Approach. Its network can be a good choice to fill up the gap between the Existing. Here we combine 3 parameters while performing the handover Load on Base Station, Distance and the Transmission Time. The results are analysed in terms of data drop respective to the speed of the mobile nodes and the distance parameter. Results shows that the presented approach has reduce the error rate of communication. Some future work can be envisaged in order to improve the handover algorithm by taking into account the requirements in terms of Quality of Service of the application, or different parameters like load on cell, network performance, security.

\section{REFERENCES}

[1] S. Balasubramaniam, J. Indulska, Vertical handover supporting pervasive computing in future wireless networks, Computer Communications 27 (8) (2004) 708-719.

[2] S. Lee, K. Sriram, K. Kim, Y.H. Kim, N. Golmie, Vertical handoff decision algorithms for providing optimized performance in heterogeneous wireless networks, IEEE Transactions on Vehicular Technology 58 (2) (2009) 865-881.

[3] E. Stevens-Navarro, V.W.S. Wong, Comparison between vertical handoff decision algorithms for heterogeneous wireless networks, in: Proceedings of the 63rd Vehicular Technology Conference (VTC'06 - Spring), Melbourne, Australia, May 2006, pp. 947-951.

[4] A.H. Zahran, B. Liang, Performance evaluation framework for vertical handoff algorithms in heterogeneous networks, in: Procee dings of the 2005 IEEE International Conference on Communications (ICC'05), Seoul, Korea, May 2005, pp. 173-178.

[5] F. Zhu, J. McNair, Optimizations for vertical handoff decision algorithms, in: Proceedings of the 2004 IEEE Wireless Communications and Networking Conference (WCNC'04), Atlanta, Georgia, USA, March 2004, pp. 867-872.

[6] W.-T. Chen, Y.-Y. Shu, Active application oriented vertical handoff in next-generation wireless networks, in: Proceedings of the 2005 IEEE Wireless Communications and Networking Conference (WCNC'05), New Orleans, Louisiana, March 2005, pp. 1383-1388. 